\title{
Performance Data from the NIST Photovoltaic Arrays and Weather Station
}

\author{
Matthew Boyd \\ National Institute of Standards and Technology, \\ Gaithersburg, MD 20899, USA \\ matthew.boyd@nist.gov
}

Data DOI: https://doi.org/10.18434/M3S67G

Key words: data acquisition; inverter; meteorology; photovoltaic; PV; solar; weather station.

Accepted: October 27, 2017

Published: November 1, 2017

https://doi.org/10.6028/jres.122.040

\section{Summary}

In July 2012, the National Institute of Standards and Technology (NIST) completed construction of three photovoltaic (PV) arrays on its Gaithersburg, MD campus. Comprehensive data acquisition systems were installed and an onsite weather station was also built to collect ancillary solar and meteorological measurements that are needed for the full characterization and modeling of the PV arrays. These datasets provide high-resolution, lowuncertainty, comprehensive PV performance and weather data for extended, continuous time periods. The creation of these datasets is fulfilling a need of the research and energy communities that few other datasets meet. Data from these systems have been collected for about three years at the time of this publication, between August 2014 and July 2017, and are being provided to the public via an online web portal for viewing and download.

The three arrays on campus use the same monocrystalline silicon module but are mounted in different configurations: the Canopy Array is mounted on east/west facing canopies over a parking lot, the Ground Array is mounted on tilted ground supports in an open field, and the Roof Array is mounted on tilted, weighted racks on a flat building roof. There is a single inverter at each array that is connected to the local grid via the NIST campus grid. The arrays are instrumented with research-grade sensors measuring various electrical, temperature, and meteorological parameters. At each array there are wind, ambient temperature, and various solar measurements being conducted. There is also a comprehensive weather station installed on a nearby building. The weather station includes instruments measuring the various solar components, spectral curves, ultraviolet (UV) light and infrared radiation (IR) energy, and a full suite of standard weather conditions. Reference modules are also installed near the weather station in the same manner and orientations as those in the arrays. These modules are maximum power tracked, with the current-voltage (I-V) curves traced every minute. Cameras capture images of these reference modules, the arrays, and the entire sky.

\section{Data Specifications}

Nearly all of the 360+ measurements at the arrays and weather station are sampled and saved every second, with 1-minute averages also saved. Exceptions are the all-sky images, which are saved every 5 seconds to 8 seconds, the module backsheet temperatures sampled every 10 seconds (but saved every 1 second), the spectroradiometer irradiance curves sampled and saved every 1 minute, and the images of the arrays saved every 5 minutes. NIST is making available 1-minute average values for the measurements and 1-hour intervals for the images during the first 
two complete calendar years of testing (2015 and 2016) via a web portal ${ }^{1}$ [1]; the 1-second instantaneous, higher frequency images, and data outside this time period are available upon request. Additional specifications are provided below.

Table 1. Basic dataset metadata.

\begin{tabular}{|l|l|}
\hline NIST Operating Unit & Engineering Laboratory; Energy and Environment Division \\
\hline Format & Text (Comma Separated Values, XML), Images (JPEG) \\
\hline Instrument & meteorological, solar, electrical, temperature, images \\
\hline Spatial or Temporal Elements & Spatial (Latitude $\left[{ }^{\circ} \mathrm{N}\right]$, Longitude [ $\left.{ }^{\circ} \mathrm{E}\right]$, Elevation [m]): \\
& Canopy array $=\left(39.1385^{\circ},-77.2155^{\circ}, 137 \mathrm{~m}\right)$ \\
& Ground array $=\left(39.1319^{\circ},-77.2141^{\circ}, 138 \mathrm{~m}\right)$ \\
& Roof array $=\left(39.1354^{\circ},-77.2156^{\circ}, 149 \mathrm{~m}\right)$ \\
& Weather station $=\left(39.1374^{\circ},-77.2187^{\circ}, 158 \mathrm{~m}\right)$ \\
\hline Data Dictionary & Temporal: 2015-01-01 through $2016-12-31$ \\
\hline Accessibility & https://www.nist.gov/file/391591 [pdf] \\
\hline License & All datasets submitted to Journal of Research of NIST are publicly available. \\
\hline
\end{tabular}

\section{Methods}

Data were sampled and saved on a 1-second basis for nearly all of the measurements, with the main exception being the resistance temperature detectors measuring the module backsheet temperature, which were sampled at a 10-second rate. All measurements are aggregated to 1-minute values, using either an average, minimum, maximum, or sum, whichever is most appropriate. The reference module maximum power voltage and current were sampled every second, but only the 1-minute aggregate values were saved. I-V curves of these reference modules were performed and saved every minute. Images of the whole sky were saved every 8 seconds, and images of the arrays were saved every 5 minutes. The timestamps associated with all measurements and images are in Eastern Standard Time (no daylight saving time), and are within 1-second of the actual time as all data loggers, cameras, and computers are synchronized to an internet time server every day just after midnight.

While every effort was made to minimize disruptions in data flow, there were times during the test phase when data were not available or particular measurements were erroneous. A log of such instances is maintained on the website where the data can be accessed. Data loss and errors were, however, kept to an extreme minimum, with these two years of data having a combined data availability greater than $99 \%$. All radiometers at the PV arrays and weather station are cleaned three times per week, with another log also kept of the precise times.

The reader is referred to the resources listed in Table 2 for details on the construction and operation of the test facilities.

Table 2. Resources for further information on the PV arrays and weather station.

\begin{tabular}{|l|l|}
\hline General Information: & $\begin{array}{l}\text { Website at https://www.nist.gov/el/energy-and-environment- } \\
\text { division-73200/heat-transfer-alternative-energy- } \\
\text { systems/photovoltaic-0 }\end{array}$ \\
\hline Design and construction specifications: & Dougherty and Boyd 2017 [2] \\
\hline Measurement approaches: & Boyd 2015 [3] Boyd 2016 [4] \\
\hline High-level performance modeling results: & Boyd 2017 [5] \\
\hline
\end{tabular}

\footnotetext{
${ }^{1}$ https://www.nist.gov/el/energy-and-environment-division-73200/heat-transfer-alternative-energy-systems/photovoltaic-0\#data
} 
The data website contains a data dictionary that includes two main sections, one for the arrays and one for the weather station. Each table has the same format, which includes the base name of the measurement channel variable, its units, a general description, the one-minute aggregate function, and an indicator of its location. The actual variable names for the 1-second instantaneous values are the base names, but those variable names for the 1-minute values are the base names appended with either an “_Avg”, “_Min”, “_Max”, or “_Sum” suffix per their aggregate function. For the variable names ending in a number in parentheses, this suffix is positioned before the parentheses. The location indicator, in the arrays section, indicates the array where this measurement was taken. For the weather station, this indicator just indicates which of the two dataloggers at the weather station took the measurement.

\section{Configuration}

The arrays were generally designed and installed to be most economical for the customer (NIST) and installer/integrator, and their configurations have not changed since their construction. A summary of the arrays is provided in Table 3.

Table 3. Summary of the NIST campus PV arrays.

\begin{tabular}{|c|c|c|c|}
\hline & Canopy & Ground & Roof \\
\hline Array Rated DC Power [kW] & 243 & 271 & 73 \\
\hline Latitude $\left[{ }^{\circ} \mathrm{N}\right]$ & 39.1385 & 39.1319 & 39.1354 \\
\hline Longitude $\left[{ }^{\circ} \mathrm{E}\right]$ & -77.2155 & -77.2141 & -77.2156 \\
\hline Elevation $^{\mathrm{a}}[\mathrm{m}]$ & 137 & 138 & 149 \\
\hline Height $^{\mathrm{b}}[\mathrm{m}]$ & 5.11 & 0.67 & 0.08 \\
\hline Tilt $\left[{ }^{\circ}\right]$ & 5 & 20 & 10 \\
\hline Azimuth [ ${ }^{\circ} \mathrm{CW}$ from N.] & 90,270 & 180 & 180 \\
\hline Number of Modules & 1032 & 1152 & 312 \\
\hline Module Manufacturer & & Sharp $^{2}$ & \\
\hline Module Model & & NU-U235F2 & \\
\hline Module Technology & \multicolumn{3}{|c|}{ monocrystalline silicon - front contact } \\
\hline Module Rated Power [W] & \multicolumn{3}{|c|}{235} \\
\hline Modules Per String & \multicolumn{3}{|c|}{12} \\
\hline Number of Source Circuits & 86 & 96 & 26 \\
\hline Number of Combiner Boxes & 7 & 7 & 4 \\
\hline Number of Inverters & 1 & 1 & 1 \\
\hline Inverter Manufacturer & \multicolumn{2}{|c|}{ PV Powered (now Advanced Energy) } & Satcon \\
\hline Inverter Model & \multicolumn{2}{|c|}{ PVP260kW } & PVS-75 480 \\
\hline Inverter Rated Power [kW] & \multicolumn{2}{|c|}{260} & 75 \\
\hline
\end{tabular}

\footnotetext{
${ }^{2}$ Certain commercial equipment, instruments, or materials are identified in this paper to foster understanding. Such identification does not imply recommendation or endorsement by the National Institute of Standards and Technology, nor does it imply that the materials or equipment identified are necessarily the best available for the purpose.
} 


\section{Disclaimers}

The data provided are those measured from the instruments and sensors associated with the NIST PV arrays and weather station. No efforts have been made to filter data or remove stray readings. Users should be aware that occasional problems occur with any sensing system and should therefore examine data for any anomalies and assess the validity of such data points. At times when no data are present from a particular instrument, an entry of "NAN" is placed in the dataset. There may be other times when data are being recorded, but erroneous connections may result in a series of missing values, or erroneous values that are inside or outside the physically possible domain. Those values have not been removed, but the user is cautioned to be aware that they may exist in the dataset.

The experiment was monitored daily to ensure that the data acquisition and PV arrays operated normally, with any problems resolved as quickly as possible. Any problems with the data acquisition systems or arrays has been recorded in a document included with the dataset. Issues with the sensors, instruments, and data acquisition systems are rare, and there are very few data gaps. However, the arrays have experienced failures and periods of maintenance sometimes lasting several weeks. There were two significant events, both occurring at the Ground array: (1) a complete removal and reinstallation of the modules, and (2) an electrical arcing event in the inverter. The first event was to refurbish the prematurely rusting support structure, and lasted 33 days between 2015-11-02 and 2015-12-04. The second event occurred on 2015-08-25, and was likely due to a loose fuse holder in the DC combiner compartment. The inverter was brought back online on 2015-09-02, and destroyed shunt measuring instruments were replaced on 2015-09-09.

\section{Impact}

It is anticipated that these datasets will be used by developers of PV modeling software for validation and tuning, and by other PV modelers during the development of new module temperature, inverter, and whole-array performance models. PV analysts will be able to better understand causes of module and balance-of-system (BOS) component failures, dynamic electrical effects from fast moving clouds and general irradiance enhancement and transitions, and the relative effect of different array orientations and configurations, to name a few examples. The high-speed monitoring and sky imaging will allow smart-grid and solar forecasting researchers to evaluate and validate short-term irradiance forecast and inverter-grid interaction models. The datasets also can be used as a reference to test and standardize modeling approaches.

\section{Acknowledgments}

The research team would like to thank the following NIST Engineering Lab (EL) staff: Tzong Hao Chen for creating the portals, Steve Barber for the storage solutions, and Steve Bannon for the web services and application deployment. Much thanks are also given to EL’s Andrew Mundy for facilitating other related IT solutions.

\section{References}

[1] Photovoltaic Testbeds. Available at https://www.nist.gov/el/energy-and-environment-division-73200/heat-transfer-alternative-energysystems/photovoltaic-0. Accessed July 31, 2017.

[2] Dougherty B, Boyd M (2017) Model (At Least) Twice, Build Once: Experiences with the Design-Bid-Build Process for Solar Photovoltaic Arrays. Journal of Solar Energy Engineering 139(3):035001. https://doi.org/10.1115/1.4036055.

[3] Boyd MT (2015) High-Speed Monitoring of Multiple Grid-Connected Photovoltaic Array Configurations. U.S. Department of Commerce, Washington, D.C., NIST Technical Note 1896. https://doi.org/10.6028/nist.tn.1896.

[4] Boyd MT (2016) NIST Weather Station for Photovoltaic and Building System Research, National Institute of Standards and Technology. U.S. Department of Commerce, Washington, D.C., NIST Technical Note 1913. https://doi.org/10.6028/nist.tn.1913.

[5] Boyd MT (2017) Comparative Performance and Model Agreement of Three Common Photovoltaic Array Configurations. Journal of Solar Energy Engineering. https://doi.org/10.1115/1.4038314.

About the author: Matthew Boyd is the associate project leader of the photovoltaic project in the Heat Transfer and Alternative Energy Systems Group in the Energy and Environment Division within the Engineering Laboratory. The National Institute of Standards and Technology is an agency of the U.S. Department of Commerce. 